\title{
Prevalence and cause of anemia in the tribal population of the Agasthyamala Biosphere Reserve forest, Western Ghats, Kerala, India
}

\author{
Authors \\ Abhijith Mohan Rajamohan, Abid Ali Khan, Amal Sebastian, Ahamed Sidheek H., \\ Karankara Malavika Menon \\ Corresponding Author
}

Dr Anish T.S

Assistant Professor, Department of Community Medicine, Medical College Thiruvananthapuram, India

\begin{abstract}
Purpose: To study the hematological profile of the tribal population in the foothills of Agasthyamala and the prevalence of anemia in the study population

Design: Cross sectional study

Materials and Methodology: The study covered covered the Tribal people of tribal sect 'Kani'. Sample size was 72. Blood collection was done and peripheral smears were prepared. Blood was analyzed using hematological analyzer. Structured questionnaire used to analyze socio-demographic factors. The data (blood parameters- WBC count, Lymphocytes, Granulocytes, RBC count, DC, Hematocrit, MCV, MCH, $M C H C, R D W$, Platelet count, MPV, PDW, PCT) received from the hematology analyzer were then compared with standard values to check for any hematological derangements. The sera of the patients with abnormalities in peripheral smears were tested for Serum ferritin, Serum Vitamin 12 and Serum LDH.

Results: Of 72 cases studied, the prevalence of anemia (according to hemoglobin concentration levels specified by $\mathrm{WHO}$ ) is 39.4\%. Significant difference was noted between hemoglobin concentration of males and females. $(p=0.02)$. The mean hemoglobin concentration in males is $13.01(S D=1.95)$ while in females is 12.11(SD=1.75). The mean hemoglobin in children is 11.14(SD=1.19). The hematological profile showed lower than normal MCV and MCH values in 11 (15.28\%) cases and $9(12.5 \%)$ of these were confirmed to be Iron Deficiency Anemia. 6 cases (8.33\%) had higher than normal MCV value and all of them (8.33\%) were confirmed to be megaloblastic anemia. 2 (2.78\%) of the cases of Megaloblastic anemia were due to Vitamin B12 deficiency. No cases of hemolytic anemia found.

Conclusion: The prevalence of anemia in the tribal population was comparable to the general population of Kerala. Females had higher prevalence as expected. No severe cases of anemia were present. The higher proportion of nutritional anemia shows that a nutritional intervention can bring about a major change.

Keywords: Anemia, Tribal Population, Iron Deficiency Anemia, Megaloblastic Anemia, Hemolytic Anemia.
\end{abstract}

\section{Introduction}

Anemia is the most prevalent nutritional deficiency in the world ${ }^{1}$. It affects all age groups but the most vulnerable populations include preschool-age children, pregnant women, and non-pregnant women of childbearing age. Globally, anemia affects 1.62 billion people, which amounts to $24.8 \%$ of the population ${ }^{2}$. National Family Health Survey statistics reveal that every second Indian woman is anemic and 
one in every five maternal deaths is directly due to anemia $^{2}$.

WHO defines anemia as a condition in which the hemoglobin $(\mathrm{Hb})$ content of blood is lower than normal as a result of deficiency of one or more essential nutrients, regardless of the cause of such deficiencies $^{3}$. As per World Health Organisation, the hemoglobin levels to diagnose anemia at sea level in $\mathrm{g} / \mathrm{l}$ is as follows ${ }^{4}$ :

\begin{tabular}{|l|c|c|c|c|}
\hline Population & Non Anemia & Mild Anemia & Moderate Anemia & Severe Anemia \\
\hline Children 6 to 59 months of age & 110 or higher & $100-109$ & $70-99$ & Lower than 70 \\
\hline Children 5 to 11 years of age & 115 or higher & $110-114$ & $80-109$ & Lower than 80 \\
\hline Children 12-14 years of age & 120 or higher & $110-119$ & $80-109$ & Lower than 80 \\
\hline $\begin{array}{l}\text { Non-pregnant women(15 years } \\
\text { of age and above) }\end{array}$ & 120 or higher & $110-119$ & $80-109$ & Lower than 80 \\
\hline Pregnant women & 110 or higher & $100-109$ & $70-99$ & Lower than 70 \\
\hline Men (15 years of age and above) & 130 or higher & $110-129$ & $80-109$ & Lower than 80 \\
\hline
\end{tabular}

The greatest burden of anemia exists in the developing world where its causes are multifactorial. The highest prevalence is in preschoolage children (47.4\%), and the lowest prevalence is in men $(12.7 \%)^{5}$. Micronutrient deficiency has been considered a prime factor and efforts to reverse the problem have been initiated in several countries in South Asia, including India as part of the millennium development goals ${ }^{6,7}$. Prevalence of anemia in all the groups is higher in India as compared to other developing countries. In India, anemia affects an estimated $50 \%$ of the population. Many studies have emphasized on prevalence of anemia among general population. This study has focused mainly on addressing the prevalence of anemia among the tribal population in Agasthyamala Biosphere Reserve forest, Western Ghats, Kerala, India

\section{Objectives}

Primary: To study the hematological profile of the tribal population in the foothills of agasthyamala

Secondary: To study the prevalence of anemia and its cause in the study population

\section{Materials and Methodology}

Study Design: Cross sectional

Study Setting: Tribal settlement at Kotoor, Kuttichal, Trivandrum.

Study period: August 10 to October 10, 2017
Study Population: Tribal people of tribal sect 'Kani' residing at the selected settlement. Pregnant women are excluded from the study

\section{Exclusion Criteria}

Pregnant women

Sampling Method: No sampling done, Census Method

Sample Size: 72 people

\section{Study Variables}

Sociodemographic variables- Age, gender, education, occupation, socio economic status.

Laboratory parameters -

1) Hematological profile and blood routine parameters.

2) Peripheral Smear

3) Serum Ferritin to confirm suspected cases of iron deficiency anemia

4) Serum Vitamin B12 to confirm suspected cases of megaloblastic anemia

5) Serum LDH to confirm suspected cases of hemolytic anemia

\section{Data Collection Tools}

Semi - structured Questionnaire

Tools for collection of blood-

Tools for smear preparation-

Hematology analyzer

Tools for analysis of serum parameters - serum ferritin, serum vitamin b12, serum LDH

\section{Data Collection Technique}

Analysis of the blood collected from the tribal population as a part of a program conducted by the Directorate of Health Services, Trivandrum. 
Blood is collected by qualified medical professionals following aseptic measures and are stored in K3EDTA coated tubes, and are later fed into a hematology analyzer

The data (blood parameters) received from the blood samples from the hematology analyzer is then compared with standard values to check for any hematological derangements

The blood picture is examined for the deviations from normal morphology by the microscopic examination of the prepared smears.

\section{Data Analysis}

Hard copies of the permissions, consents, and results of hematological parameters will be kept with the primary researchers.

Data will be entered in excel sheets and analyzed using appropriate statistical software.

All quantitative variables will be expressed as mean and standard deviation and all qualitative variables will be expressed as proportion.

Significance level of $95 \%$ Confidence Interval will be determined apriori for all the analysis.

\section{Results}
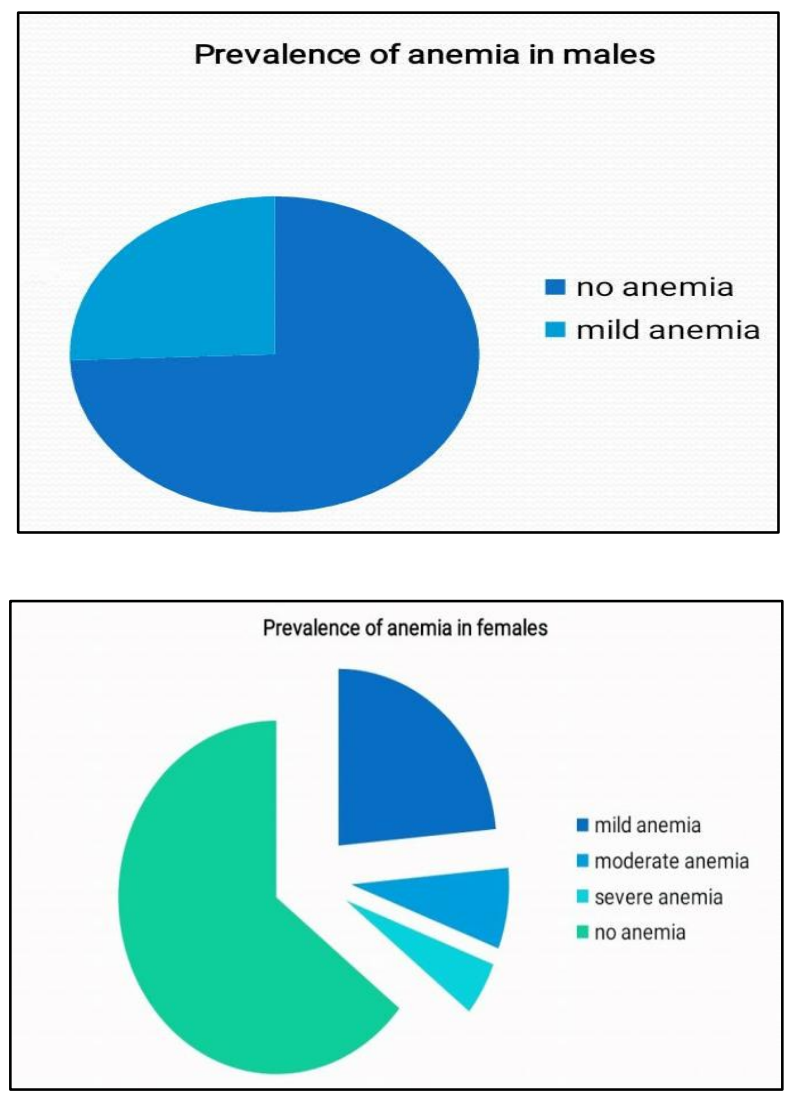

\section{Prevalence of anemia in children}

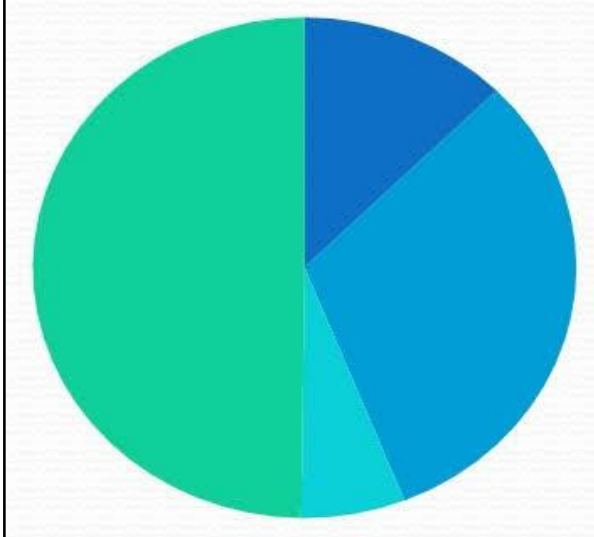

mild anemia

moderate anemia

severe anemia

no anemia

\section{Distribution of Hemoglobin Concentration}

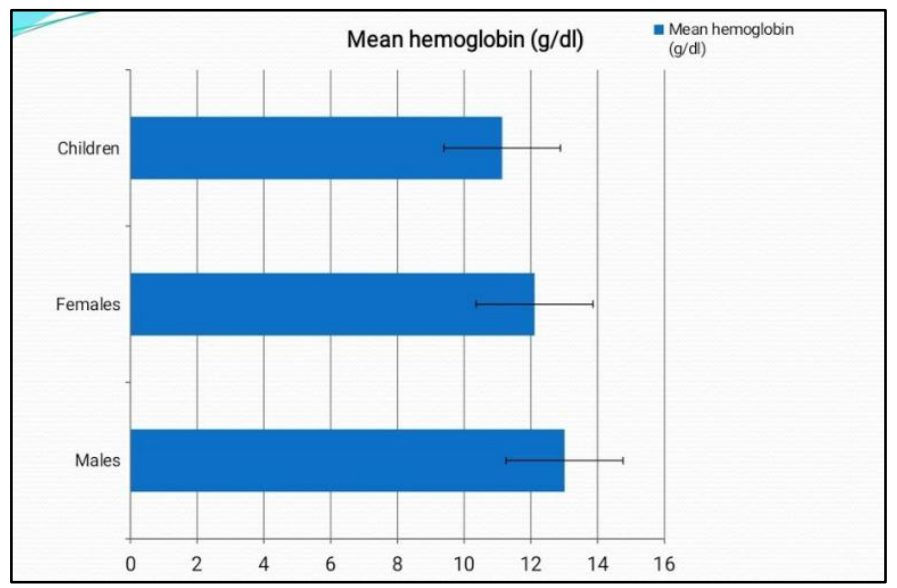

\section{Discussion}

In this cross-sectional study, 72 people of sect 'Kani' residing at the tribal settlement at Kotoor, Kuttichal, Trivandrum were studied to assess their hematological profile and their prevalence of anemia and its causes.

Of 72 cases studied, the prevalence of anemia (according to hemoglobin concentration levels specified by WHO) was $39.4 \%$. Among males, only mild anemia was prevalent with a prevalence of $25.6 \%$. (74.4\% were found to be normal). In females, prevalence of mild anemia is $23.5 \%$, moderate anemia is $7.4 \%$ and severe anemia is 4.9\%. Among children, prevalence of mild, moderate and severe anemia is $12.2 \%, 31.5 \%$ and $6.2 \%$ respectively. Significant difference was noted between hemoglobin concentration of males and females. $(p=0.02)$. The mean hemoglobin conc. in males is $13.01(\mathrm{SD}=1.95)$ while in females 
is $12.11(\mathrm{SD}=1.75)$. The mean hemoglobin in children is $11.14(\mathrm{SD}=1.19)$.

When the cause of anemia was analysed, the hematological profile showed 11 cases with lower than normal MCV and $\mathrm{MCH}$ values. The peripheral smears pointed to picture of microcytic hypochromic anemia. Serum Ferritin done on these 11 cases confirmed Iron deficiency anemia in 9 cases (lower than normal ferritin values according to Advanced Clinical Research Lab). 6 cases had higher than normal MCV value and peripheral smear showed picture of megaloblastic anemia. 2 cases had lower than normal value for Serum Vitamin B12 and pointed to 'Megaloblastic anemia due to Vitamin B12 deficiency'. No cases of hemolytic anemia found. LDH values were within normal limits.

\section{Conclusion}

- The prevalence of anemia in the tribal population was comparable to general population of Kerala.

- Females had higher prevalence as expected. No severe anemia cases were present. These show the effective public health service utilization by the tribal community and the efficiency of the PHC in making the services accessible even in the interiors of the biosphere reserve.

- The hematological profile showed 11 cases with lower than normal $\mathrm{MCV}$ and $\mathrm{MCH}$ values. And the peripheral smears pointed to picture of microcytic hypochromic anemia.

- Serum Ferritin done on these 11 cases confirmed Iron deficiency anemia in 9 cases

- 6 cases had higher than normal MCV value and peripheral smear showed picture of megaloblastic anemia.

- 2 cases had lower than normal value for Serum Vitamin B12 and pointed to 'Megaloblastic anemia due to Vitamin B12 deficiency'.
- The cases were reported to PHC and treatment was initiated. The higher proportion of nutritional anemia shows that a nutritional intervention can bring about a major change.

\section{Reference}

1. Stoltzfus RJ. Defining iron-deficiency anemia in public health terms: a time for reflection. The Journal of nutrition. 2001 Feb 1;131(2):565S-7S.

2. Kaur K. Anaemia 'a silent killer'among women in India: Present scenario. European Journal of Zoological Research. 2014;3(1):32-6.

3. World Health Organization. Nutritional anaemias: report of a WHO scientific group [meeting held in Geneva from 13 to 17 March 1967].

4. https://www.who.int/vmnis/indicators/hae moglobin.pdf

5. WHO, WHO global database on anaemia / Edited by Bruno de Benoist, Erin McLean, Ines Egli and Mary Cogswell, 2008. http://whqlibdoc.who.int/publications/200 8/9789241596657_eng.pdf retrieved on July February 6th , 2014.

6. Freedman I, Wirth ME, Waldman R, Chowdhury M, Rosenfeld A. Millenium Project Task Force 4: Child Health Interim Report, NY Millenium Project 2004.

7. Osrin D, Vaidya A, Shrestha Y, Baniya RE, Manandhar DS, Adhikari RK, Filteau S, Tomkins A, Costello AM. Effects of antenatal multiple micronutrient supplementation on birth weight and gestational duration in Nepal: Doubleblind, randomized controlled trial. Lancet 2005;365:955-962. 